\section{An eclectic look at fungal genetics}

\author{
Essential Fungal Genetics \\ D Moore and L-AN Frazer \\ Springer-Verlag, New York; 2002. \\ $€ 79.95$, hardback. ISBN 0-387-96367-1
}

Heredity (2004) 92, 595. doi:10.1038/sj.hdy.6800470

\section{Reviewed by JM Kelly}

Essential Fungal Genetics contains a collection of very different chapters, some on very basic general genetics and other excellent chapters on specific aspects of fungal genetics and biology, which form a not entirely satisfactory whole because it is difficult to identify the target reader - is it the professional mycologist, the geneticist, the mushroom enthusiast or the undergraduate mycologist? In the preface, the authors indicate, 'we hope that it will be a user-friendly guide that can be used as a supplement to general genetics texts'. To achieve the authors' objective, it would have been preferable to omit much of the extensive coverage of basic genetics included in the book, which is not sufficiently illustrated to be clear to a novice geneticist, on the understanding that the reader will consult one of the wide range of excellent general genetics texts where necessary. This would free up space to include a more complete discussion of important specific fungal genetics topics that are glossed over, such as fungal plant pathogen interactions, and fungal biotechnology, as well as more comprehensive referencing in each chapter. Nevertheless, each of the specialist chapters is comprehensive and authoritative, and an excellent and readily digested resource for the advanced reader.

The first chapter is an excellent general introduction to the diversity and evolutionary relationships of members of the fungal kingdom, and introduces the concept of microbial fungi as model systems for studying eukaryotic biology. The second chapter looks at genome interactions, highlighting vegetative incompatibility and mating-type systems, with an excellent discussion of recent genetic and molecular genetic advances in a variety of fungal species that have led to an understanding of the mechanisms in some cases. A mature understanding of genetic concepts would be required to fully appreciate this specialist chapter, and having read thus far, I assumed that the book was aimed at an advanced graduate student or research scientist. Thus, I was surprised to find that two chapters covering the basic general genetics concepts followed this advanced chapter. The third chapter ranges from Mendelism through mutation, mutagenesis and chromosomal rearrangements to RFLP's site-directed mutagenesis and gene disruption. The fourth chapter covers classical genetic analysis including complementation, segregation and the detection of linkage. Fungal examples and experimental detail are included, and these chapters are comprehensive, but the lack of figures sufficiently clear to illustrate key concepts will be a problem for an undergraduate or general audience.

The fifth and sixth chapters are excellent in that they show the power of using fungi in genetic analysis when advantage is taken of particular facets of fungal life cycles, especially random and ordered asci to analyse meiosis, and the ability of some haploid fungi to form stable diploids to study mitotic recombination, and the haploidization of these diploids to follow whole chromosomes. There is a detailed discussion of the analysis of aberrant segregation ratios in tetrads, leading to a model to understand the molecular basis of recombination and gene conversion. These topics are frequently not comprehensively covered in modern general genetics textbooks, and this chapter will provide a very welcome supplement for students of fungal genetics.

Chapters seven and eight again cover mainly generic basic concepts dealing with the physical analysis of DNA across the spectrum from DNA polymorphic marker detection to pulse field electrophoretic karyotyping to DNA sequencing, including a detailed description of methodology in some cases. Again, these areas are comprehensively covered in most general genetics textbooks, and could be omitted.

Chapter nine begins as a primer of population and evolutionary genetics, which is perhaps better dealt with in more user-friendly general genetics texts, and then continues to fungal examples, which are of considerable interest and would have made an excellent specialist chapter. The regulation of fungal metabolism, differentiation and development is considered in chapter ten, which covers an enormous amount of information with a considerable degree of success for the informed reader. Both of these chapters would be even more useful with more extensive specific referencing.

The strength of the book is that the information is authoritative and wide ranging, and I am quite sure that I will consult it often, and recommend chapters to graduate students. However, it seems that the book falls between audiences with its mix of very basic genetics chapters, which are not as clearly presented and well illustrated as many genetics texts for the beginner, and very advanced specialist fungal genetics topics, which are not as comprehensively referenced as might be expected by the specialist.

JM Kelly

School of Molecular and Biomedical Science, Molecular Life Sciences Building, The University of Adelaide, North Terrace, Adelaide 5005, Australia E-mail: joan.kelly@adelaide.edu.au 